\title{
Mode-Locked Dual-Wavelength Heterolasers for Terahertz Generation via Intracavity Wave Mixing
}

\author{
A.A. Belyanin ${ }^{a}$, V.V. Kocharovsky ${ }^{a, b}$, \\ Vl.V. Kocharovsky ${ }^{b}$, V.A. KukUshikin ${ }^{b *}$, A.V. Andrianov $^{b}$, \\ V.YA. Aleshinin ${ }^{c}$ And A.A. Dubinov ${ }^{c}$ \\ ${ }^{a}$ Physics Department, Texas A\&M University, College Station, TX 77843, USA \\ ${ }^{b}$ Institute of Applied Physics of RAS \\ 46 Ulyanov st., 603950 N. Novgorod, Russia \\ ${ }^{c}$ Institute for Physics of Microstructures of RAS \\ GSP-105, 603950 N. Novgorod, Russia
}

\begin{abstract}
It is shown that the mid/far-infrared (IR) and $\mathrm{THz}$ pulse generation via intracavity difference-frequency mixing in quantum-well dual-wavelength heterolasers can be rather efficient under mode-locking regime for one or both lasing fields even at room temperature. In a simple model we derive an explicit formula for intensity of the generated IR or $\mathrm{THz}$ pulse and find that this method is capable of producing picosecond pulses at $\approx 1 \mathrm{GHz}$ repetition rate with the peak power of the order of $1 \mathrm{~W}$ and $\leq 0.2 \mathrm{~mW}$ at $10 \mu \mathrm{m}$ and $50 \mu \mathrm{m}$ wavelengths, respectively.
\end{abstract}

PACS numbers: 42.55.Px, 42.65.Ky

\section{Introduction}

The generation of mid- to far-infrared (IR) and $\mathrm{THz}$ powerful radiation is an important problem in the modern optics and laser physics due to its necessity for fundamental studies and various applications. Though there are many different techniques to produce intense coherent emission in this frequency range, a well-known nonlinear optical process of difference-frequency (DF) mixing of two strong driving fields remains one of the most efficient and promising ways for its generation. Such schemes are divided roughly into two categories. The first of them involves DF mixing of external laser fields in second-order nonlinear media or quantum well (QW) heterostructures [1-3]. Unfortunately, these methods as a

*corresponding author; e-mail: vakuk@appl.sci-nnov.ru 
rule have a rather low down conversion efficiency, in particular because of strong driving field depletion via one or multi-photon absorption.

Another way of obtaining IR and THz radiation is based on DF mixing of laser modes in the same cavity where they are generated [4]. Such devices do not suffer from driving field depletion since it is mitigated by laser gain. They are based on the parametric IR or THz generation which can work at room temperature in $\mathrm{CW}$ regime, as opposed to $\mathrm{THz}$ quantum cascade lasers that require cryogenic cooling. In addition, intracavity mixing devices can be pumped by current injection, which makes corresponding IR and $\mathrm{THz}$ sources much cheaper and more compact than those with external optical pumping. Current pumping, however, has also a negative outcome as it leads to a high free-carrier density in the active region and, as a consequence, strong IR and $\mathrm{THz}$ field absorption which rapidly increases with wavelength growing. Being added to diffraction leakage and modal overlap both of which deteriorate with wavelength increasing, the resulting nonlinear conversion efficiency is typically rather low.

In the present paper we consider pulsed intracavity DF generation when one or both near-infrared driving fields are generated in the mode-locking regime. This obviously enhances all nonlinear effects and can potentially provide higher peak output power of the IR or $\mathrm{THz}$ radiation as compared to the $\mathrm{CW}$ regime. Moreover, in the case of both optical fields being generated in the mode-locking regime [5], this can enhance an average DF generation power as well.

\section{Model of a quantum-well heterolaser}

As an example, let us consider an $\mathrm{Al}_{x} \mathrm{Ga}_{1-x} \mathrm{As}$ QW heterostructure consisting of two adjacent wells made of two 5-8 nm thin layers separated by a thin spacer $(\approx 30 \mathrm{~nm})$ and incorporated into a core of an AlGaAs and plasmon waveguide [6]. Further, we shall consider a simple structure with one heavy-hole (1) and two electron (2 and 3) states in the left asymmetric QW and one hole (4) and one electron (5) state in the right one, so that the transition $3 \rightarrow 1$ is resonant with $5 \rightarrow 4$ one (Fig. 1). Of course, all the following considerations can be easily suited for the situation when two hole and one electron levels in the left QW are used. The interband transitions correspond to $\lambda \approx 1 \mu \mathrm{m}$, whereas intersubband transitions lie at $\lambda \approx 10 \mu \mathrm{m}$ for the electronic levels and even at longer wavelengths $\gtrsim 70 \mu \mathrm{m}$ for the hole states [4]. It is well known that for asymmetric QWs (e.g., step-like or coupled double QWs) all three transitions $2 \rightarrow 1,3 \rightarrow 2,3 \rightarrow 1$ can have large matrix elements at $\approx 0.3-1 \mathrm{~nm}$ in the near-IR range and $\approx 1-5 \mathrm{~nm}$ in the far-infrared range [3].

Two optical fields $\boldsymbol{E}_{1}, \boldsymbol{E}_{2}$ with central frequencies $\omega_{1}, \omega_{2}$ and longitudinal wave numbers $k_{1}, k_{2}$ are generated in the left and right QWs at $2 \rightarrow 1$ and $5 \rightarrow 4$ transitions in the $\mathrm{CW}$ or mode-locking regimes [5], respectively, with injection current pumping providing the necessary inversion. Due to resonant coupling on the $3 \rightarrow 2$ transition in the left QW and non-resonant interaction through secondorder semiconductor bulk nonlinearity these fields create pulses of long-wavelength 


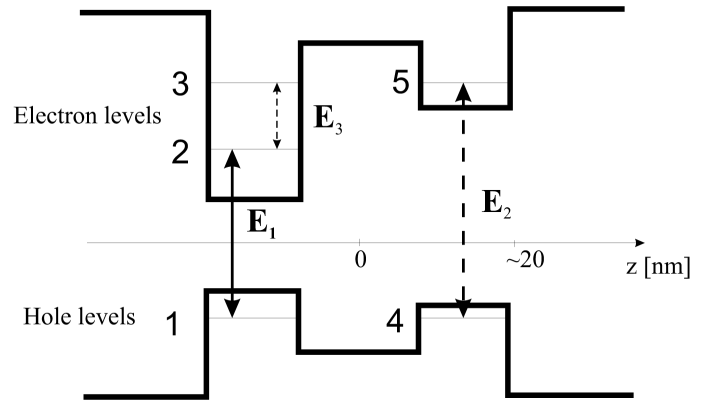

Fig. 1. The scheme of electron (hole) levels and transitions involved in the IR or $\mathrm{THz}$ pulse generation in a QW heterolaser.

(IR or $\mathrm{THz}$ ) polarization with the central longitudinal wave number $k_{3}=k_{2}-k_{1}$ and frequency $\omega_{3}=\omega_{2}-\omega_{1}$. This polarization, in turn, produces an IR or $\mathrm{THz}$ electromagnetic pulsed field $\boldsymbol{E}_{3}$ via excitation of corresponding cavity modes.

Then, employing for the present situation the standard density matrix formalism [4] and cavity excitation theory [7], we derive a formula for the longwavelength output field intensity at the central plane of the waveguiding structure, $I_{\text {out3 }}$, as a function of output optical field intensities in the same plane, $I_{\text {out } 1,2}$. The values $I_{\text {out } 1,2,3}$ are related to intensities $I_{1,2,3}$ just under the end facet of the waveguide core by a simple formula $I_{\text {out } 1,2,3}=\mathrm{T}_{1,2,3} \mathrm{I}_{1,2,3}$, where $T_{1,2,3}$ are the corresponding transmission coefficients at the facet mirror. So we get

$$
I_{\text {out3 }}=\frac{16 \pi T_{3} n_{3} \omega_{3}^{2} l_{3}^{2} l_{\mathrm{coh}}^{2}}{T_{1} T_{2} c^{3}\left(l_{3}^{2}+l_{\mathrm{coh}}^{2}\right)}\left|\frac{c \varsigma_{3}\left(\boldsymbol{d}_{23} \tilde{\boldsymbol{e}}_{-k_{3}}\right) f}{2 \pi \hbar n_{3} \varsigma_{1} \omega_{1} \gamma l_{1}}+\pi \chi_{\mathrm{eff}}\right|^{2} I_{\mathrm{out} 1} I_{\mathrm{out} 2},
$$

where $n_{i}=\operatorname{Re}\left[\sqrt{\epsilon_{1}\left(\omega_{i}\right)}\right]$ and $l_{i} \equiv c /\left(n_{i}\left|\omega_{k_{i}^{\prime \prime}}\right|\right) i=1,2,3$, are the refraction indices and the absorption lengths for all the three fields, $\omega_{k_{i}} \equiv \omega_{k_{i}}^{\prime}+\mathrm{i} \omega_{k_{i}}^{\prime \prime}$ - the complex eigenfrequencies of the driving and generated fields, $l_{\text {coh }}=c /\left(n_{3}\left|\omega_{3}-\omega_{k_{3}}^{\prime}\right|\right)=$ $c v_{2} /\left(n_{3} \omega_{3}\left|v_{2}-c / n_{3}\right|\right)$ - an effective coherence length, $v_{2}$ - the optical group velocity, $\gamma$ - a polarization relaxation time $(\approx 0.1 \mathrm{ps}), f$ - a numerical factor of the order of unity,

$$
\varsigma_{1,3}=\frac{S_{\text {l-well }}}{\int \varepsilon\left(\omega_{1,3}\right) \tilde{\boldsymbol{e}}_{-k_{1,3}} \tilde{\boldsymbol{e}}_{k_{1,3}} \mathrm{~d} S},
$$

where $S_{\text {l-well }}$ is the area of the left QW, $\varepsilon\left(\omega_{1,3}\right)$ - the structure dielectric functions at optical and IR or THz frequencies, $\tilde{\boldsymbol{e}}_{k_{1,3}}$ - the complex amplitudes of the optical and long-wavelength cavity modes, $\boldsymbol{e}_{k_{1,3}} \equiv \boldsymbol{e}_{k_{1,3}}(x=0) \exp \left(\mathrm{i} k_{1,3} x\right) \equiv$ $\tilde{\boldsymbol{e}}_{k_{1,3}} \exp \left(\mathrm{i} k_{1,3} x\right), \boldsymbol{e}_{-k}$ is determined through $\boldsymbol{e}_{k}$ according to [7], $\boldsymbol{d}_{23}$ - the dipole moment of the $3 \rightarrow 2$ transition (which is chosen to be real),

$$
\chi_{\mathrm{eff}} \equiv \frac{\int \sum_{i, l, m=1}^{3} \chi_{i l m} \tilde{e}_{-k_{3} i} \tilde{e}_{k_{2} l} \tilde{e}_{k_{1} m}^{*} \mathrm{~d} S}{\int \epsilon\left(\omega_{3}\right) \tilde{\boldsymbol{e}}_{k_{3}} \tilde{\boldsymbol{e}}_{-k_{3}} \mathrm{~d} S},
$$

where $\chi_{i l m}$ is the second-order semiconductor bulk nonlinear susceptibility. 


\section{Power estimates}

Let us estimate the power of the generated IR or THz DF pulses. We consider first a particular example of an IR signal at a wavelength $\lambda_{3} \approx 10 \mu \mathrm{m}$ generated by two optical fields with the wavelengths $\lambda_{1} \approx 0.8 \mu \mathrm{m}$ and $\lambda_{2} \approx 0.73 \mu \mathrm{m}$, typical of $\mathrm{Al}_{x} \mathrm{Ga}_{1-x} \mathrm{As}$ heterostructures. For $l_{1} \approx 0.1 l_{3} \approx 10 \mu \mathrm{m}$ we get a peak power of $\approx 0.1 \mathrm{~W}$ (which corresponds to an average power of $0.65 \mathrm{~mW}$ at $\approx 1 \mathrm{GHz}$ repetition rate and 5 ps pulse duration). For the longer wavelengths of the output field lying beyond the reststrahlen region of the strong absorption and dispersion of GaAs $/ \mathrm{Al}_{x} \mathrm{Ga}_{1-x} \mathrm{As}, \lambda_{3} \gtrsim 50 \mu \mathrm{m}$, we get estimates of the peak pulse and average powers at, say, $\lambda_{3}=50 \mu \mathrm{m}$ to be $8.6 \mu \mathrm{W}$ and $43 \mathrm{nW}$, respectively, for $l_{1} \approx 10 \mu \mathrm{m}$ (for $\lambda_{3}=120 \mu \mathrm{m}$ the analogous values are $0.37 \mu \mathrm{W}$ and $1.85 \mathrm{nW}$, correspondingly). Such a quick drop of the output power is a result of the rapid enhancement of nonresonant losses $l_{3}(50 \mu \mathrm{m}) \approx 0.04 l_{3}(10 \mu \mathrm{m}), l_{3}(120 \mu \mathrm{m}) \approx 0.02 l_{3}(10 \mu \mathrm{m})$ and the decrease in the ratio $\omega_{3} / \omega_{1}$ in Eq. (1) with wavelength growth.

It is interesting to note that though the linear absorption of the longwavelength field is greatly enhanced near the transverse optical phonon frequency (which corresponds to $\approx 35 \mu \mathrm{m}$ for GaAs), the second-order nonlinear coefficient also rises rapidly near this point, where it becomes $\approx 30$ times higher than at $10 \mu \mathrm{m}$ $[8,9]$. Then, taking into account the DF mode spreading to be much larger than the thickness of the highly absorptive confining layer, it can be concluded that the long-wavelength field absorption at $\approx 35 \mu \mathrm{m}$ is still defined by non-resonant losses due to free carriers. Therefore, in the case of large enough $l_{1}=100 \mu \mathrm{m}$ when the bulk nonlinearity can give an appreciable contribution to the generated IR or $\mathrm{THz}$ power, it may turn out advantageous to generate the DF field near the phonon resonance where the peak pulse and average powers can be of the order of $3 \mu \mathrm{W}$ and $15 \mathrm{nW}$ for $l_{1}=100 \mu \mathrm{m}$, i.e. increased by more than one order of magnitude in comparison with estimates for $\lambda_{3}=50 \mu \mathrm{m}$ and the same $l_{1}=100 \mu \mathrm{m}$.

\section{Various designs and methods of increasing the nonlinear IR signal}

It is important to mention that final Eq. (1) for the long-wavelength field pulse shape and intensity still holds qualitatively true when both driving fields, $\boldsymbol{E}_{1}$ and $\boldsymbol{E}_{2}$, are pulsed [5]. In this situation the increase in $\boldsymbol{E}_{1}$ field in the pulse peak compared to its $\mathrm{CW}$ value can lead to a significant growth of the output IR or $\mathrm{THz}$ intensity. In such a regime in the experiments similar to that of [5] we would have two, say, 5 ps optical pulses with the peak powers of $24 \mathrm{~W}$ each, i.e., get an increase by a factor of 20 in the peak intensity of the $\boldsymbol{E}_{1}$ pulse in comparison with its $\mathrm{CW}$ value. Then, e.g., for an $\mathrm{Al}_{x} \mathrm{Ga}_{1-x} \mathrm{As} \mathrm{QW}$ heterostructure with $l_{1} \approx 10 \mu \mathrm{m}$, we could get the peak power of the long-wavelength field of order $2.6 \mathrm{~W}$ (an average power of $13 \mathrm{~mW}$ ) at $\lambda_{3}=10 \mu \mathrm{m}, 0.2 \mathrm{~mW}$ (an average power of $1 \mu \mathrm{W}$ ) at $\lambda_{3}=50 \mu \mathrm{m}$ and of $7.4 \mu \mathrm{W}$ (an average power of $37 \mathrm{nW}$ ) at $\lambda_{3}=120 \mu \mathrm{m}$. We stress the fact that average IR or $\mathrm{THz}$ output power is greatly increased if one 
implements this mode-locking regime for both optical fields of the nonlinear-mixing dual-wavelength heterolaser.

In some cases it is not technologically easy to grow two or more adjacent QWs with controllable parameters or even one QW with two close electron or hole levels. In this regard, let us note that there exists a simple method consisting in employing the so-called "butt-joint" scheme [10], i.e., two QW lasing heterostructures either of which is responsible for the generation of just one driving field, $\boldsymbol{E}_{1}$ or $\boldsymbol{E}_{2}$. These devices are butt-connected so that a $\mathrm{CW}$ or pulsed optical field generated in one structure passes into another and vice versa. To avoid strong interband absorption, one needs to send a lower-frequency optical field through a laser generating a higher-frequency optical field, where the DF mixing occurs. Of course, in this case we do not take advantage of the resonant nonlinear quantum coherence between QW subbands, but in the case of moderate optical losses an appreciable part of the output IR or $\mathrm{THz}$ power can be determined by the bulk nonlinearity contribution and, therefore, a butt-joint scheme can be rather efficient in this case.

\section{Acknowledgments}

The work was partly supported by AFOSR (grants No. FA-9550-05-1-0360 and FA-9550-06-1-0338), NSF (grants No. ECS-0501537, EEC-0540832, R39863, and ECS-0547019), the Research Corporation (award No. RI0986), CRDF (grant No. RUE1-2657-NN-05), ISTC (grant No. 2293), Presidium RAS (programs "Low-dimensional quantum structures" and "Electromagnetic waves of the THz range"), Department of Physical Sciences of RAS (program "Semiconductor lasers"), RFBR-BRFBR (grant No. 02-02-81036), RFBR (grants No. 05-02-17525, 07-02-00486) and the Council for support of the leading scientific schools in Russia (grant No. 4588.2006.2).

\section{References}

[1] Y.R. Shen, The Principles of Nonlinear Optics, Wiley, New York 1984.

[2] A.A. Belyanin, V.V. Kocharovsky, Vl.V. Kocharovsky, D.S. Pestov, M.O. Scully, J. Mod. Opt. 51, 2523 (2004).

[3] Semiconductor Lasers, Ed. E. Kapon, Academic Press, San Diego 1999.

[4] A.A. Belyanin, F. Capasso, V.V. Kocharovsky, Vl.V. Kocharovsky, M.O. Scully, Phys. Rev. A 63, 053803 (2001).

[5] P.J. Delfyett, in: Ultrafast Lasers: Technology and Applications, Ed. M.E. Fermann, A. Galvanauskas, G. Sucha, Marcel Dekker, Inc., New York 2001.

[6] V. Berger, C. Sirtori, Semicond. Sci. Technol. 19, 964 (2004).

[7] L.A. Vainshtein, Electromagnetic Waves, Sov. Radio, Moscow 1988 (in Russian).

[8] C. Flytzanis, Phys. Rev. B 6, 1264 (1972).

[9] A.A. Afonenko, V.Ya. Aleshkin, A.A. Dubinov, Semicond. Sci. Technol. 20, 357 (2005).

[10] A.A. Biryukov, V.Ya. Aleshkin, S.M. Nekorkin, V.V. Kocharovsky, Vl.V. Kocharovsky, B.N. Zvonkov, M.O. Scully, J. Mod. Opt. 52, 2323 (2005). 